\title{
The role of autophagy in the pathogenesis of glycogen storage disease type II (GSDII)
}

\author{
AC Nascimbeni ${ }^{1}$, M Fanin ${ }^{1}$, E Masiero ${ }^{2}$, C Angelini ${ }^{1,3}$ and M Sandri ${ }^{\star \star 2,4}$
}

Regulated removal of proteins and organelles by autophagy-lysosome system is critical for muscle homeostasis. Excessive activation of autophagy-dependent degradation contributes to muscle atrophy and cachexia. Conversely, inhibition of autophagy causes accumulation of protein aggregates and abnormal organelles, leading to myofiber degeneration and myopathy. Defects in lysosomal function result in severe muscle disorders such as Pompe (glycogen storage disease type II (GSDII)) disease, characterized by an accumulation of autophagosomes. However, whether autophagy is detrimental or not in muscle function of Pompe patients is unclear. We studied infantile and late-onset GSDII patients and correlated impairment of autophagy with muscle wasting. We also monitored autophagy in patients who received recombinant $\alpha$-glucosidase. Our data show that infantile and late-onset patients have different levels of autophagic flux, accumulation of p62-positive protein aggregates and expression of atrophy-related genes. Although the infantile patients show impaired autophagic function, the lateonset patients display an interesting correlation among autophagy impairment, atrophy and disease progression. Moreover, reactivation of autophagy in vitro contributes to acid $\alpha$-glucosidase maturation in both healthy and diseased myotubes. Together, our data suggest that autophagy protects myofibers from disease progression and atrophy in late-onset patients. Cell Death and Differentiation (2012) 19, 1698-1708; doi:10.1038/cdd.2012.52; published online 18 May 2012

Macroautophagy, hereafter referred to as autophagy, is a highly conserved homeostatic process for lysosomemediated degradation of cytoplasmic components, including damaged organelles and toxic protein aggregates. ${ }^{1}$

Being the largest protein reservoir in the body, muscle serves as a source of amino acids that can be used for energy production by various organs during catabolic periods. Protein degradation in all mammalian cells, including skeletal muscle, is controlled by the two major proteolytic systems, the ubiquitin-proteasome and the autophagy-lysosome. Both systems are under FoxO regulation and their excessive activation induces severe muscle loss. ${ }^{2}$ The central role of lysosomes in myofiber homeostasis is highlighted by the presence of a group of muscle disorders characterized by alterations in lysosomal function, that ultimately affects the cargo delivery (autophagy). Glycogen storage disease type II (GSDII) is due to a defect in lysosomal acid $\alpha$-glucosidase (GAA), ${ }^{3}$ Danon disease is caused by the lack of lysosomeassociated membrane protein 2 (lamp2) ${ }^{4}$ and X-linked myopathy with excessive autophagy (XMEA) ${ }^{5}$ is triggered by mutations in an essential assembly chaperone of the V-ATPase, the principal mammalian lysosomal proton pump complex. ${ }^{6}$ Furthermore, chloroquine-mediated inhibition of lysosome function induces a well-known myopathy. ${ }^{7}$
GAA is the enzyme responsible for the lysosomal hydrolysis of glycogen to glucose. Without GAA, glycogen delivered to the lysosome accumulates, resulting in different clinical phenotypes. In the severe infantile-onset form, known as Pompe disease, little or no GAA activity causes hypotonia, feeding difficulties and cardiomyopathy, leading to death from cardiac failure within the first year of life. Partial enzyme defects induce late-onset forms that are mainly characterized by progressive skeletal muscle weakness, while cardiac muscle is spared. The animal model of Pompe disease, GAA-knockout (GAA-KO) mice, mainly recapitulates the infantile or early childhood forms of this disease, due to the complete lack of the enzyme.

The presence of large, glycogen-filled lysosomes is a hallmark of GSDII, but it has also been reported that the pathological features of this disorder in skeletal muscle involve failure of autophagosomal turnover and massive autophagic buildup.,9 However, genetic suppression of autophagy in skeletal muscles significantly reduced the glycogen burden in GAA-KO mice ${ }^{10}$ but did not improve clinical signs such as kyphosis, muscle wasting and decreased life span. ${ }^{11}$ Our recent data show that inhibiting autophagy specifically in muscle tissue causes muscle weakness and features of myofiber degeneration. ${ }^{12}$ Defining

\footnotetext{
1Department of Neurosciences, University of Padova, Biomedical Campus 'Pietro d'Abano', Padova, Italy; ${ }^{2}$ Dulbecco Telethon Institute at Venetian Institute of Molecular Medicine (VIMM), Padova, Italy; ${ }^{3}$ RCCS S. Camillo, Venice, Italy and ${ }^{4}$ Department of Biomedical Science, University of Padova, Padova, Italy

${ }^{*}$ Corresponding author: M Sandri, Department of Biomedical Science, Dulbecco Telethon Institute at Venetian Institute of Molecular Medicine (VIMM), University of Padova, Via G. Orus 2, Padova 35129, Italy. Tel: +39 049 7923258; Fax: +39 049 7923250; E-mail: marco.sandri@unipd.it

Keywords: atrophy; autophagy; glycogen storage disease; Pompe disease; MuRF1

Abbreviations: GSDII, glycogen storage disease type II; GAA, acid $\alpha$-glucosidase; p62/SQSTM1, p62/sequestosome1; FoxO, forkhead box 0; Lamp2, lysosomalassociated membrane protein 2; XMEA, X-linked myopathy with excessive autophagy; V-ATPase, vacuolar-type H + -ATPase; GAA-KO, acid $\alpha$-glucosidase-knockout; PGC1 $\alpha$, peroxisome proliferator-activated receptor $\gamma$ coactivator 1- $\alpha$; ERT, enzyme replacement therapy; LC3, microtubule-associated protein 1 light chain 3; LC3II, microtubule-associated protein 1 light chain $3 \beta$; CSA, cross-sectional area; BECN1, Beclin 1; Bnip3, Bcl-2/adenovirus E1B 19 kDa interacting protein 3; MuRF1, musclespecific RING finger protein 1; M6P, mannose-6-phosphate; mTOR, mammalian target of rapamycin; NF $\kappa$ B, nuclear factor $\kappa$-light-chain-enhancer of activated B cells; NRF2, nuclear factor (erythroid-derived 2)-like 2; PKC, protein kinase C; GAPDH, glyceraldehyde 3-phosphate dehydrogenase; H\&E, hematoxylin and eosin stain; IF, immunofluorescence; PAS, periodic acid-Schiff; Cav3, caveolin-3; HBSS, Hank's-balanced salt solution

Received 28.9.11; revised 27.2.12; accepted 20.3.12; Edited by E Baehrecke; published online 18.5.12
} 
the role of autophagy in GSDII and its relationship with muscle loss is critical for understanding the disease pathogenesis, for developing new therapies and for improving efficacy of enzyme replacement therapy (ERT).

To understand the relationship between autophagy and disease progression, we have monitored autophagy system in muscle biopsies of early and late-onset GSDII. Moreover, we used patients' and healthy control myotubes to corroborate the findings obtained from muscle biopsies. Our data support the hypothesis that autophagy is helpful for myofiber survival in late-onset forms of GSDII by facilitating GAA maturation. Importantly, impairment of autophagy contributes to myofiber atrophy and muscle weakness.

\section{Results}

Monitoring autophagy and atrophy in GSDIl patient muscle. Our previous work demonstrated that the accumulation of p62/SQSTM1 into protein aggregates is a hallmark of impaired autophagic flux in myofibers. ${ }^{12}$ Thus, we have used p62-positive inclusions as a marker to monitor autophagy impairment in muscle tissue from GSDII patients (Supplementary Table S1) and controls and have correlated the presence of protein aggregates with vacuolization and myofiber atrophy.

Infantile-onset patients. First, we quantified the protein levels of LC3 and p62, two markers of autophagosome formation and clearance, respectively, by western blotting (Figure 1a; Supplementary Figure S1a). Except for an ERTtreated case (8668), Pompe patients had a massive increase in lipidated LC3 and p62 protein. Morphological analyses showed the presence of large vacuoles and p62-positive protein aggregates in the vast majority of fibers (Figure 1b). Quantification of p62 inclusion-positive versus negative as well as vacuolated versus normal fibers confirmed the abundance of muscle cells with these features. ERT greatly reduced the proportion of fibers with these abnormalities in patient 8668 (Figure 1b) as well as p62 protein and LC3II band (Figure 1a), suggesting that autophagy flux was restored. We then asked whether myofiber size is affected

a

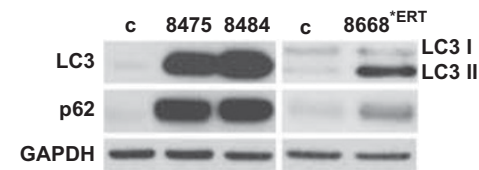

b

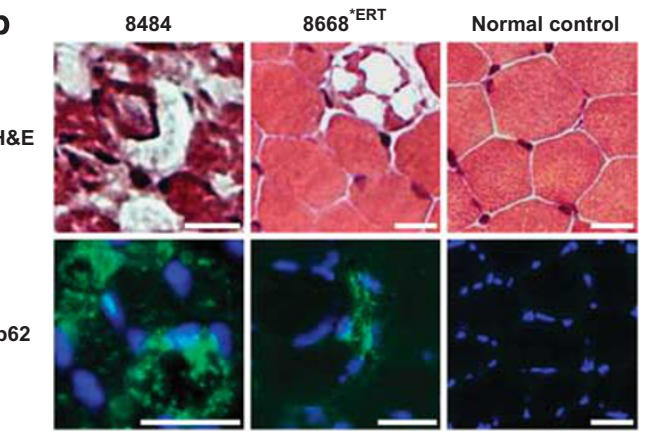

C

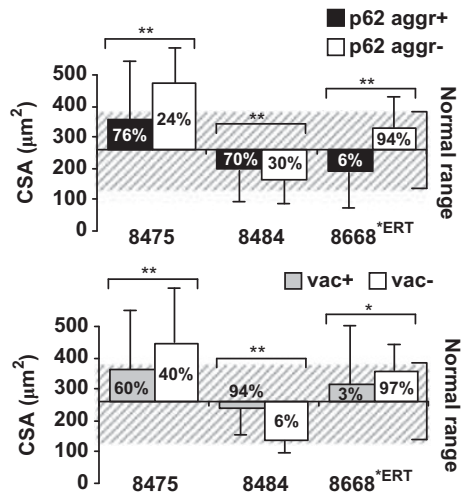

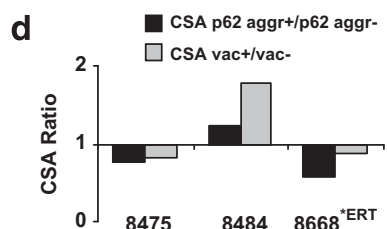

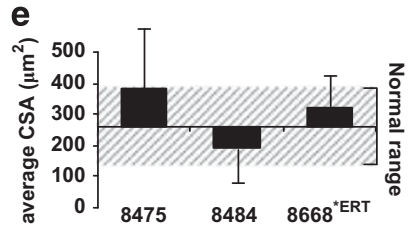

f
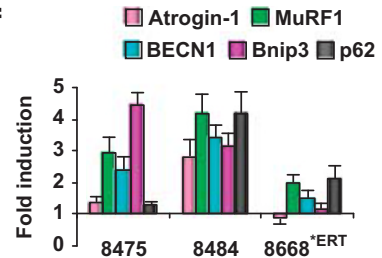

Figure 1 Characterization of autophagy and atrophy in infantile-onset GSDII patients. (a) Immunoblot analysis of LC3, p62 and the loading control (GAPDH). c: agematched healthy control; numbers: patient identifier; "ERT: patient subjected to ERT prior to biopsy. (b) H\&E staining and p62 immunohistochemistry: example of two patients and a normal control. Bar: $20 \mu \mathrm{m}$. (c) CSA of fibers showing p62 aggregates (p62 aggr + ) compared with fibers without p62 aggregates (p62 aggr - ) and vacuolated (vac + ) compared with non-vacuolated (vac - ) fibers, relative to healthy age- and sex-matched controls (mean CSA of controls: $258 \pm 124 \mu \mathrm{m}^{2}$; dashed box: normal CSA range). The percentages of p62 aggregate-positive, negative, vacuolated and non-vacuolated fibers are reported in the bars. ${ }^{\star \star} P<0.0001 ;{ }^{\star} P<0.05$. $n>200$ fibers measured. (d) CSA ratio of p62 aggregate-positive versus negative and vacuolated versus non-vacuolated fibers. When the size of fibers containing p62 aggregates is smaller than the negative fibers it will result in a ratio below 1. Identical consideration applies to vacuolated fibers. (e) Average CSA of all the muscle fibers. (f) qRT-PCR analysis of the atrophyrelated genes and of p62. The fold induction is compared with the age-matched control and normalized to GAPDH 
when autophagy (p62 aggregates) or lysosomes (vacuoles) are compromised. Comparison of cross-sectional area (CSA) of p62 aggregate-positive versus negative and vacuolated versus non-vacuolated fibers showed an increased atrophy in p62 aggregate-positive or vacuolated fibers in two of three patients (Figure 1c). The patient 8484 had an inverse pattern, possibly because huge vacuoles were present that altered myofiber structure and caused abnormal muscle fiber morphology (Figure 1b). Interestingly, p62 aggregates did not always correlate with vacuolization, suggesting that the presence of vacuoles does not always mean autophagy impairment. Moreover, the ERT-treated patient almost completely reverted the abnormal features being the p62 inclusion-positive and vacuolated fibers reduced to 6 and $3 \%$ of total fibers, respectively (Figure 1c). Indeed the majority of fibers are free of protein aggregates and vacuoles (Figure 1b) and show almost normal glycogen deposits, whereas untreated infantile patients had a generalized impairment of glycogen clearance (Supplementary Figure S2).

To understand the relationship between autophagy failure and vacuolization in muscle loss, we determined the ratio between the CSA of p62 aggregate-positive versus p62 aggregate-negative and of vacuolated versus non-vacuolated fibers. A ratio below 1 indicates that the altered fibers are more atrophic than surrounding normal ones. The ratio of p62-aggregate positive was always lower than the ratio of vacuolated fibers meaning that autophagy impairment is detrimental for muscle mass maintenance and contributes to muscle atrophy per se (Figure 1d). The comparison of the average CSA suggests that patient 8484 is the most affected one (Figure 1e), showing small and vacuolated fibers. Autophagy impairment was demonstrated also by the massive autophagic buildup observed by the immunostaining for p62 and LC3 (Supplementary Figure S3a).

Loss of muscle mass is controlled by a transcriptional program that requires activation of a subset of atrophy-related genes (atrogenes). ${ }^{13}$ Among atrogenes, some are rate limiting factors of the ubiquitin-proteasome and autophagylysosome systems. The upregulation of these factors is required to increase protein breakdown. Thus, their expression was monitored in patients. In addition, p62 transcript was studied and correlated to protein level and to aggregates. Interestingly, the ubiquitin ligases Atrogin-1 and MuRF1, and the autophagy-related genes Bnip3, Beclin1 and p62 were significantly upregulated in Pompe patients (Figure 1f). The ERT-treated patient showed lower atrophy-related gene expression.

Childhood-onset patients. Immunoblotting analysis showed an increase of LC3II in both patients. However, the most severely affected (3096) showed dramatic accumulation of lipidated LC3 and p62, suggesting an impairment of autophagic flux. Importantly, the increased level of p62 is not due to a transcriptional upregulation (Figure 2f). The other patient did not display any accumulation of p62 protein despite an increase of LC3II (Figure 2a; Supplementary Figure S1b). Morphological analysis confirmed the biochemical results (Figure $2 \mathrm{~b}$ ). Indeed, the most compromised child showed p62-positive aggregates and vacuolization in $30 \%$ of fibers, while the milder child hold only $15 \%$ of altered fibers
(Figures 2c and d). P62 aggregate-positive and vacuolated fibers were more atrophic than p62 aggregate- and vacuolenegative fibers in both patients. Consistently with the morphology, average CSA showed atrophy in the severely affected patient 3096 and was normal in the milder one (Figure 2e). Gene expression analyses showed an induction of the autophagy and ubiquitin-proteasome genes only in the more compromised patient (Figure 2f).

Adult-onset patients. Unlike infantile patients, adultonset patients show heterogeneous clinical severity, disease progression, muscle wasting and vacuolization ${ }^{14}$ (Figure 3b). Immunoblot analysis confirmed this heterogeneity, showing different degrees of autophagic impairment. Lipidated LC3 and p62 proteins range from elevated to normal levels (Figure 3a; Supplementary Figure S1c). Interestingly, accumulation of p62 did not always correlate with an increase in LC3II. Quantification of myofibers with p62-positive aggregates enabled us to establish a correlation between autophagic impairment and myofiber atrophy. The proportion of fibers with protein aggregates strongly correlated with but did not completely match the number of vacuolated fibers (Supplementary Figure S3b). Indeed p62-positive aggregates were also detected in non-vacuolated fibers (Supplementary Figure S4). The percentage of myofibers containing p62 inclusions or vacuoles ranged from 80 to $2 \%$ (Figure $3 c$ ). Consistently with previous data, most of the patients showed a reduction in myofiber size in p62 aggregate-positive and vacuolated fibers when compared with surrounding normal ones. Indeed, the CSA ratios were below one, suggesting that the altered fibers are atrophic. Interestingly, autophagyimpaired fibers are more atrophic than vacuolated ones (Figure 3d).

Gene expression analyses strengthened the correlation between muscle atrophy (Figure $3 e$ ) and MuRF1 expression (Figure 3f), the ubiquitin ligase involved in thick filament degradation. Indeed the two male patients with the lowest CSA $(7329,8557)$ showed the highest induction of MuRF1 and of other atrogenes, while the patient who displayed a normal autophagic flux and myofiber size (6373) had the lowest MuRF1 level.

A similar trend was observed in the female patients. Those with the most compromised autophagic flux, detected by $p 62$ aggregates, also displayed the highest MuRF1 expression. The female patient with normal myofiber size (5639) (Figure 3e) showed no accumulation of LC3II (Figure 3a), the lowest percentage of vacuolated- and p62 aggregatepositive fibers (Figure 3c), and depicted a suppression of MuRF1 (Figure 3f). Interestingly, myofiber atrophy and p62 aggregate-positive fibers showed a correlation with MuRF1 expression but not with Atrogin-1 induction (Figures $3 e$ and f).

Disease progression in GSDIl patients and autophagy impairment. To better elucidate the contribution of autophagy impairment to disease progression, three adult female patients underwent a second biopsy after 6 or 9 years. Comparison of these two time points showed a different degree of autophagy impairment revealed by accumulation of LC3 and p62 proteins (Figure 4a; Supplementary Figure S1d). The concomitant transcriptional upregulation of p62 in 
a
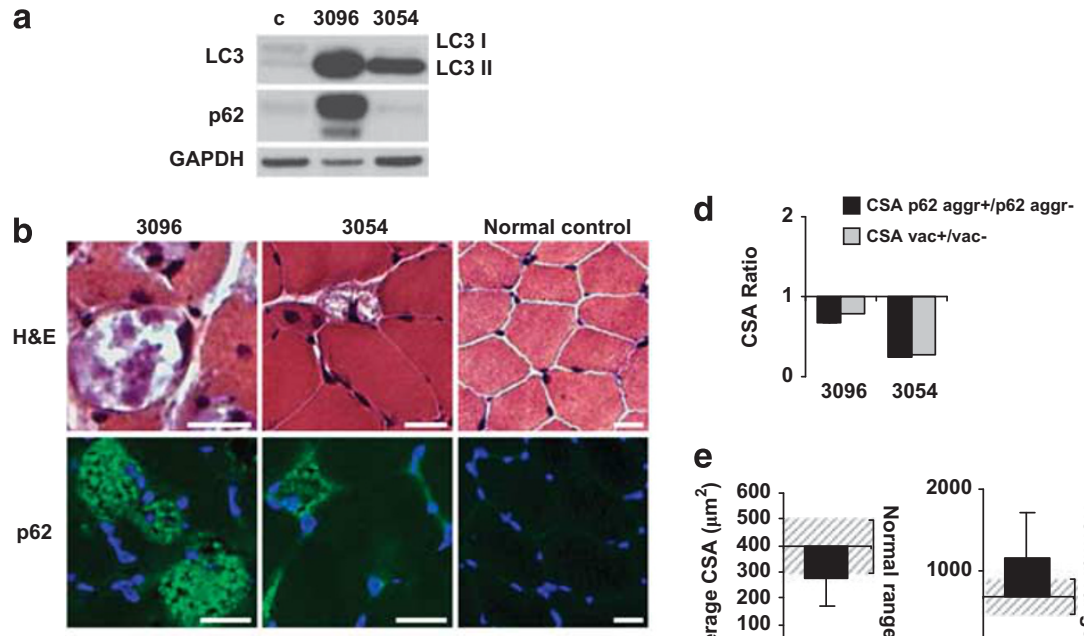

C
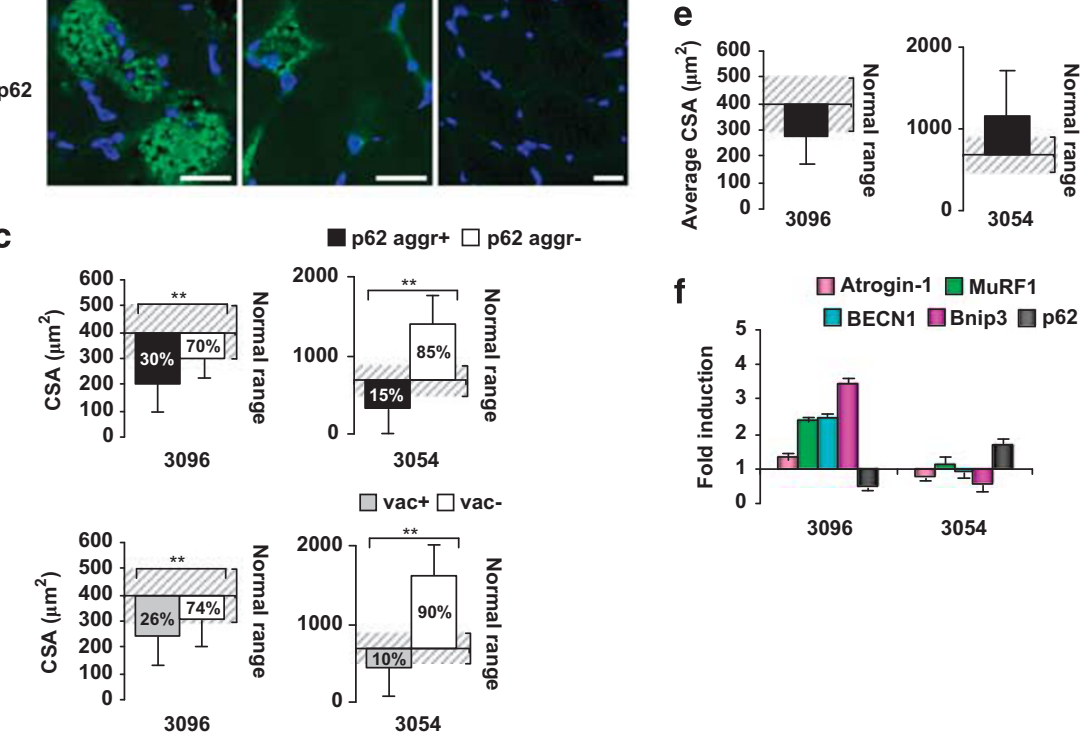

Figure 2 Characterization of autophagy and atrophy in childhood-onset GSDIl patients. (a) Immunoblot analysis of LC3, p62 and the loading control (GAPDH). (b) H\&E staining and p62 immunohistochemistry. Bar: $20 \mu \mathrm{m}$. (c) CSA of fibers showing p62 aggregates (p62 aggr + ) compared with fibers without p62 aggregates (p62 aggr -) and vacuolated ( $\mathrm{vac}+$ ) compared with non-vacuolated (vac - ) fibers, relative to healthy age- and sex-matched controls (4-year-old controls mean CSA: $395 \pm 79 \mu \mathrm{m}^{2} ; 7$-yearold controls mean CSA: $681 \pm 193 \mu \mathrm{m}^{2}$; dashed box: normal CSA range). The percentages of p62-positive, negative, vacuolated and non-vacuolated fibers are reported in the bars. ${ }^{\star \star} P<0.0001 . n>200$ fibers measured. (d) CSA ratio of p62-aggregates positive versus negative and vacuolated versus non-vacuolated fibers. (e) Mean of CSA of total myofibers. (f) qRT-PCR analysis. Fold induction is compared with the age-matched control and normalized to GAPDH

these patients (Figure 4f) results in a difficult interpretation of western blot data. However, morphological analyses of vacuolated and p62 aggregate-positive fibers revealed that only one patient (5639) showed a time-dependent increase of abnormalities, confirming a progression of the disease (Figures $4 \mathrm{~b}$ and c). Patient 5639 displayed an almost normal myofiber morphology, muscle mass and gene expression profile at the time of the first biopsy (Figures $4 b$, e and $f$ ). Therefore, this patient was perfect to study the correlation between disease progression and alterations in autophagic flux. The morphometric analyses showed a trend of decrease of CSA in all the patients that well correlates with disease progression (Figures $4 \mathrm{c}-\mathrm{e}$ ). The most striking case was subject 5639, who had a $60 \%$ decrease in CSA during 6 years of disease progression. P62 aggregate-positive and vacuolated myofibers increased from 2 to $40 \%$ in subject 5639 , whereas the other two patients remained unchanged. The dramatic muscle loss that occurred in patient 5639 was consistent with the induction of MuRF1, while the milder patients showed minor or no changes of MuRF1 expression (Figure 4f). Conversely, there was no time-dependent increase of Atrogin-1 expression in patient 5639 while it was significantly upregulated in the second biopsy of the other two patients. These findings are crucial because correlate muscle atrophy to MuRF1 but not Atrogin-1 expression.

ERT increases muscle mass and restores autophagy. An infantile (8484) and an adult (8557) patient were treated for 16 and 6 months, respectively, with recombinant GAA. After treatment, a second biopsy was taken to evaluate autophagy, aggregates, vacuoles and muscle atrophy and was compared with the pre-treatment biopsy. The adult patient responded better to GAA therapy showing a decrease of LC3 lipidation, p62-positive aggregates and fiber vacuolization (Figures 5a-c; Supplementary Figure S1e) that resulted in normalization of fiber size (Figure 5e) and atrogenes expression (Figure 5f). The infant started therapy at 8 months old, an age that has been described as being too late for successful recombinant GAA uptake. ${ }^{15}$ Indeed, the second biopsy of this patient showed higher p62 protein content without any transcriptional upregulation (Figures $5 \mathrm{a}$ 
a

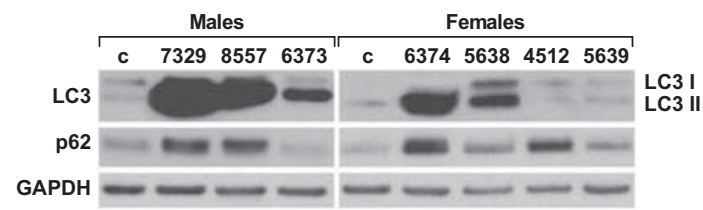

b
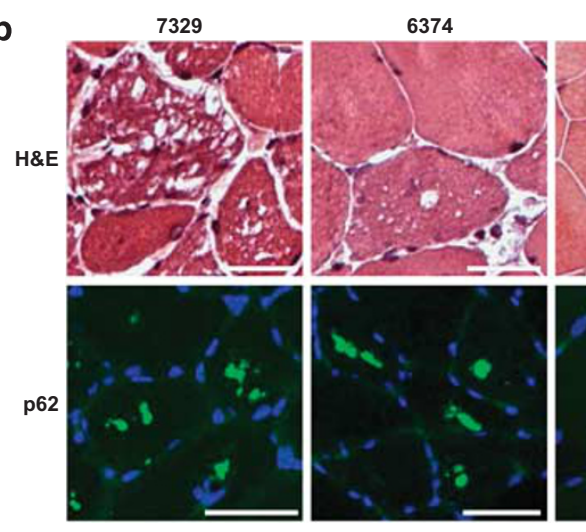

4512
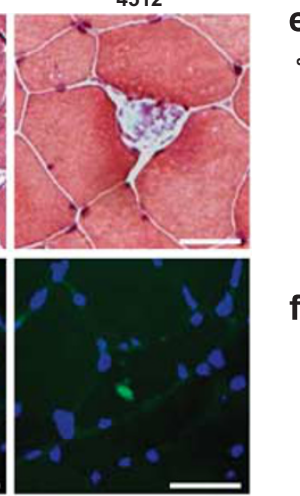

C
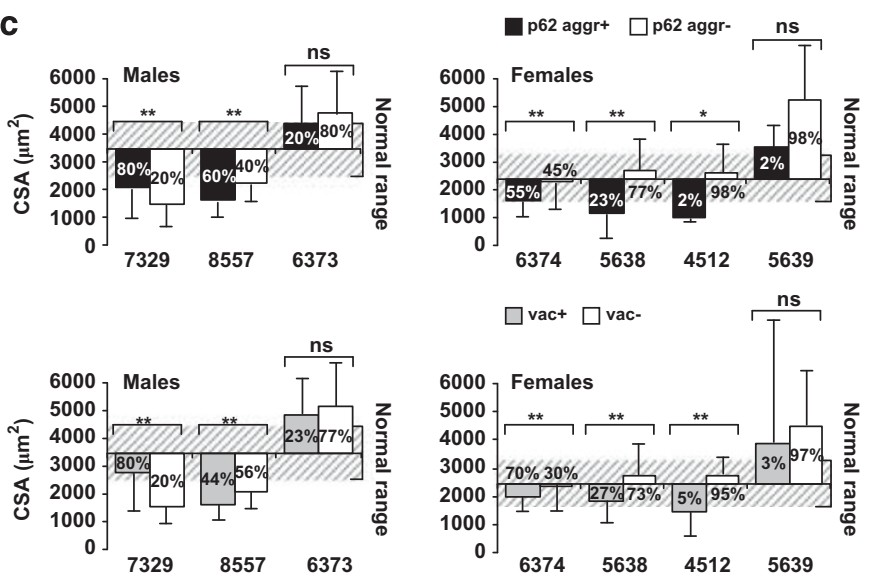

d
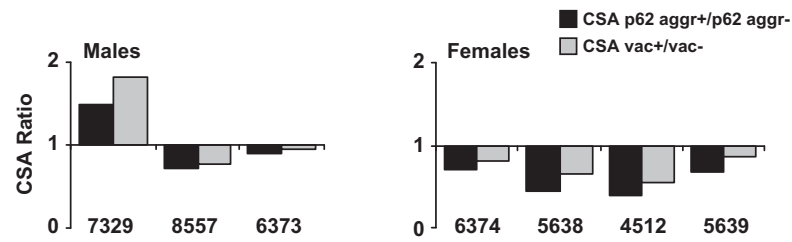

e

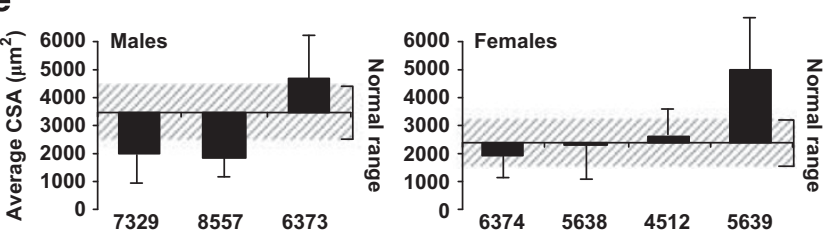

f 88 A Atrogin-1 $\square$ MuRF1 $\square$ BECN1 $\square$ Bnip3 $\square$ p62

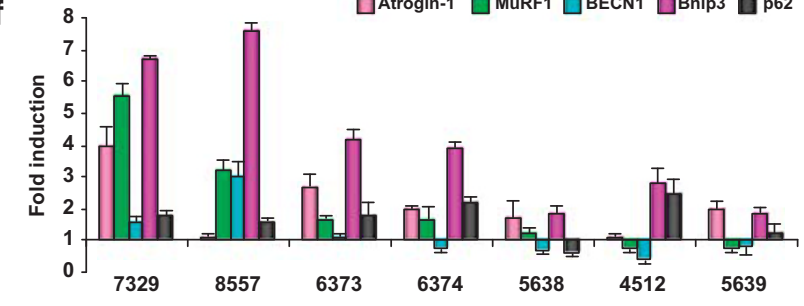

Figure 3 Characterization of autophagy and atrophy in adult-onset GSDII patients. (a) Immunoblot analysis of LC3, p62 and the loading control (GAPDH). (b) H\&E staining and p62 immunohistochemistry. Bar: $40 \mu \mathrm{m}$. (c) CSA of fibers showing p62 aggregates (p62 aggr + ) compared with fibers without p62 aggregates (p62 aggr - ) and vacuolated (vac +) compared with non-vacuolated (vac - ) fibers, relative to healthy age- and sex-matched controls (female controls mean CSA: $2419 \pm 786 \mu \mathrm{m}^{2}$; male controls mean CSA: $3446 \pm 950 \mu \mathrm{m}^{2}$; dashed box: normal CSA range). The percentages of p62-positive, negative, vacuolated and non-vacuolated fibers are reported in the bars. ${ }^{\star \star} P<0.0001 ;{ }^{*} P<0.05$; ns: not significant. $n>200$ fibers measured. (d) CSA ratio of $p 62$-aggregates positive/negative and vacuolated/non-vacuolated fibers. (e) Total average CSA. (f) qRT-PCR analysis. Fold induction is compared with the age-matched control and normalized to GAPDH

and f), suggesting a persistent autophagic impairment despite GAA treatment. Importantly, the percentage of p62 aggregate-positive fibers increased from 70 to $98 \%$ while the percentage of vacuolated ones was already high before ERT (94\%) and did not change after (98\%) (Figure 5c).

This patient had no detectable GAA mature forms and instead elevated levels of the inactive 110 precursor (Figure 5a). ERT induced the appearance of mature $76 \mathrm{kDa}$ GAA even if at very low level. The ratio of p62 aggregatepositive versus negative fibers and vacuolated versus non-vacuolated fibers was higher than 1, probably because of the large size of the vacuoles and aggregates, which dramatically disrupt myofiber structure and size (Figures $5 \mathrm{c}$ and d). The adult patient showed low level of mature GAA that increased after rhGAA (recombinant human GAA) administration (Figure 5a).
GAA treatment in the adult patient greatly reduced the number of p62-positive and vacuolated fibers from 60 to $34 \%$ and from 44 to $35 \%$, respectively (Figures $5 \mathrm{~b}$ and c). Interestingly, the CSA ratio of p62 aggregate-positive versus negative did not significantly change, while the ratio of vacuolated fibers improved (Figure 5d), confirming the hypothesis that vacuoles do not always mirror autophagic impairment. Thus, the fibers that hold autophagy flux disruption maintained the atrophic phenotype. Unlike the infantile patient, ERT in adult patient was also able to enhance glycogen clearance in muscle, significantly decreasing glycogen storages (Supplementary Figure S2). Consistently with morphology, therapy in adult patient greatly increased muscle mass (Figure 5e) and downregulated most of the atrogenes (Figure 5f). The CSA values in the infantile patient looked like there was an amelioration after ERT. However, we emphasize 
a

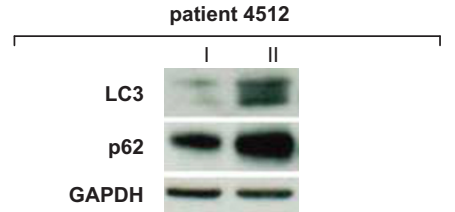

b
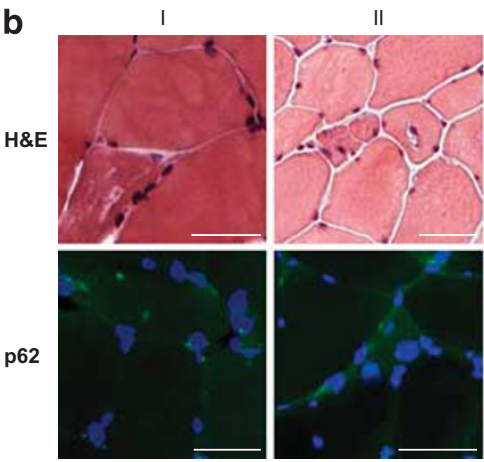

C

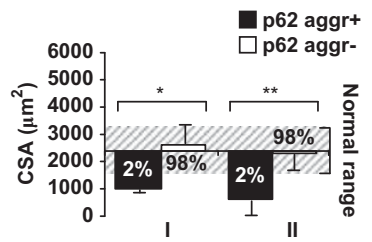

$\square$ vac+ $\square$ vac-

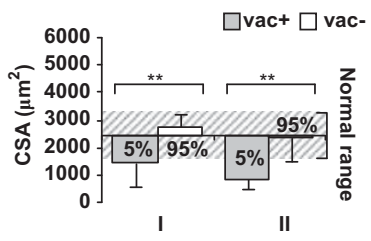

d

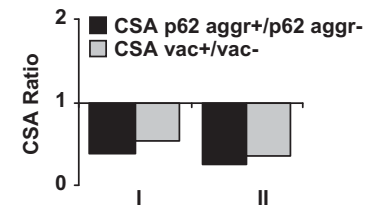

e

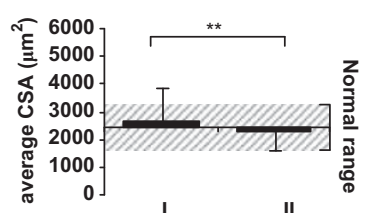

f

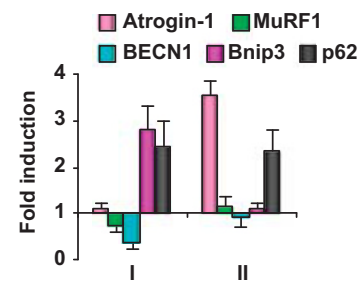

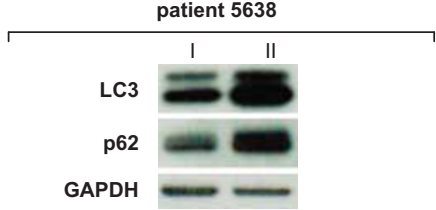
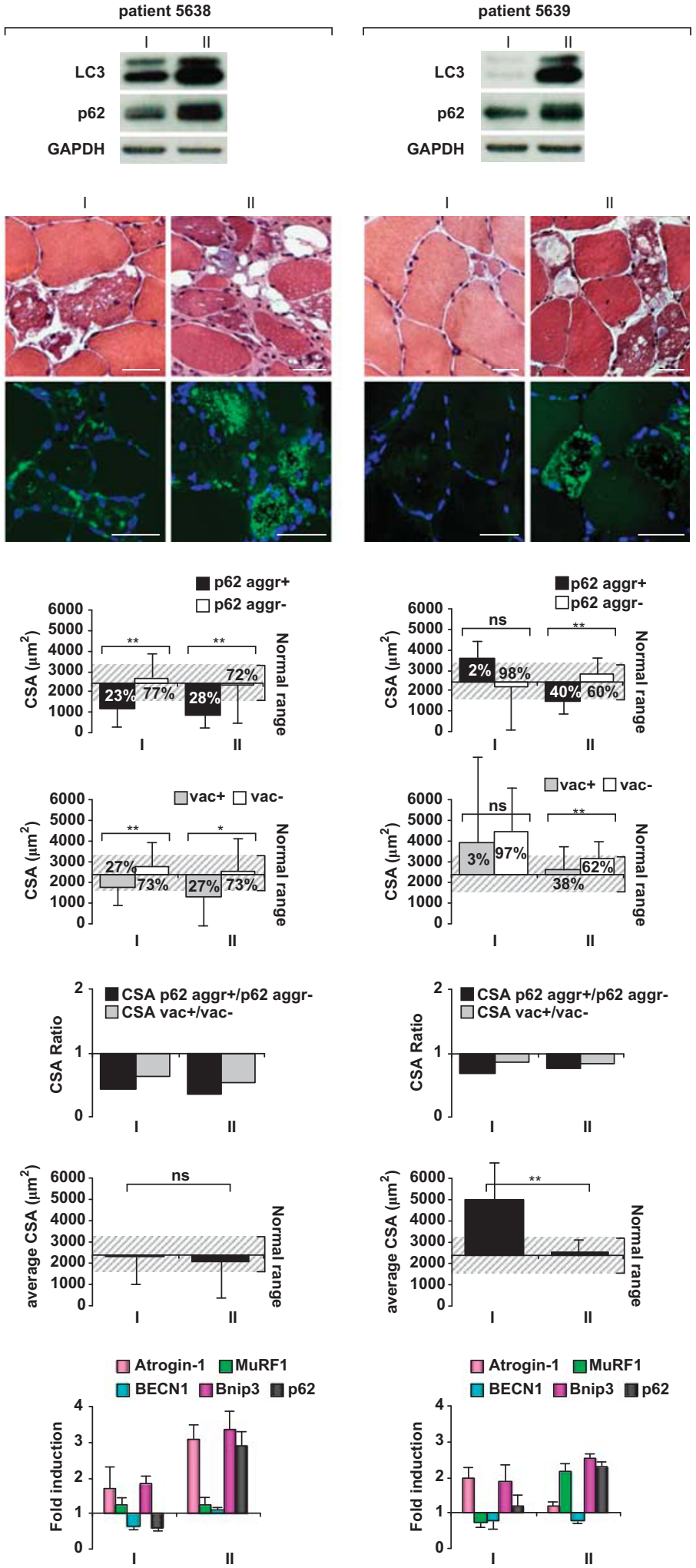

Figure 4 Comparison of different staged biopsies. (a) Immunoblot analysis of LC3, p62 and the loading control (GAPDH). I: first biopsy; II: second biopsy. (b) H\&E staining and p62 immunohistochemistry. Bar: $40 \mu \mathrm{m}$. (c) CSA of fibers showing p62 aggregates (p62 aggr + ) compared with fibers without p62 aggregates (p62 aggr - ) and vacuolated (vac + ) compared with non-vacuolated (vac - ) fibers, relative to healthy age- and sex-matched controls (mean CSA for female controls: $2419 \pm 786 \mu \mathrm{m}^{2} ;$ dashed box: normal CSA range). The percentages of p62-positive, negative, vacuolated and non-vacuolated fibers are reported in the bars. ${ }^{\star \star} P<0.0001 ;{ }^{*} P<0.05 ; n s:$ not significant. $n>200$ fibers measured. (d) CSA ratio of p62-aggregates positive/negative and vacuolated/non-vacuolated fibers. (e) Average CSA of all the muscle fibers. ${ }^{* *} P<0.0001$; ns: not significant. (f) qRT-PCR analysis. Fold induction is compared with the age-matched control and normalized to GAPDH 
a

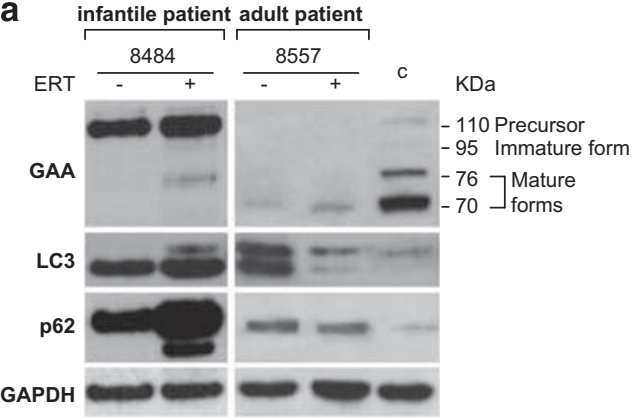

b

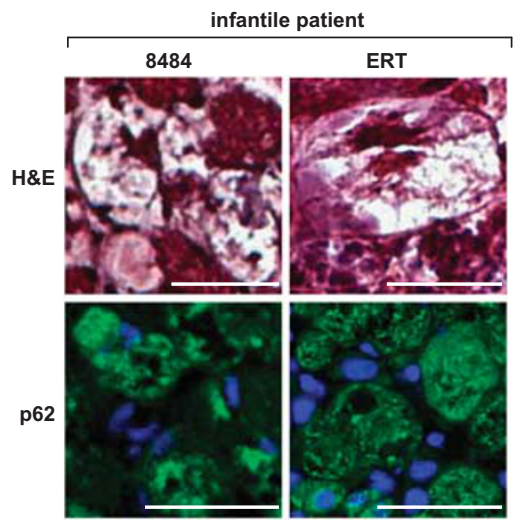

adult patient

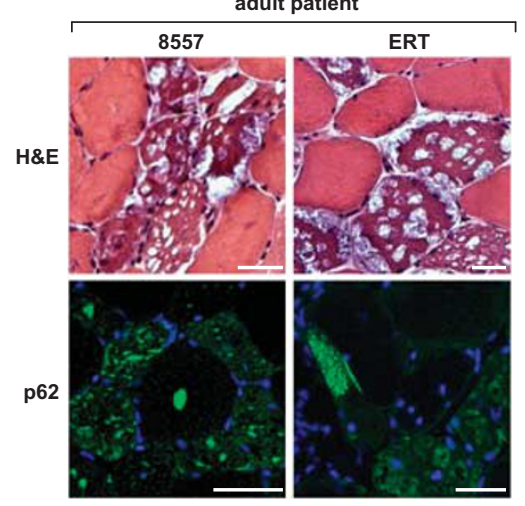

c
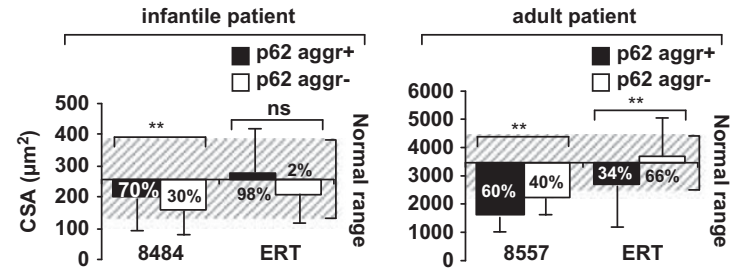

$\square$ vac+ $\square$ vac-
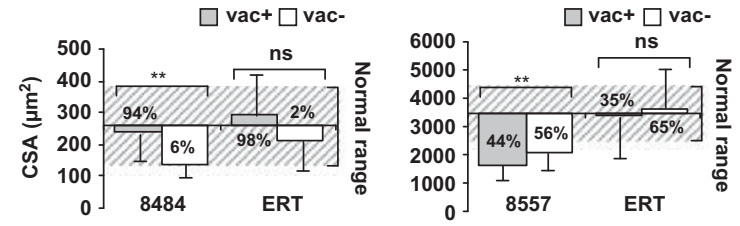

d
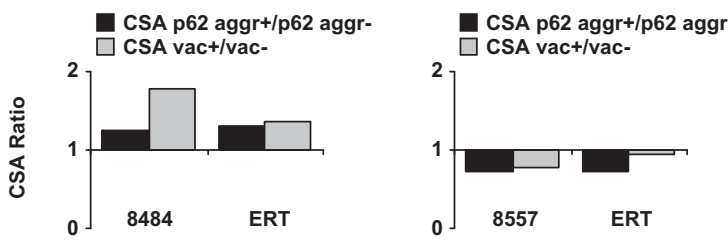

e
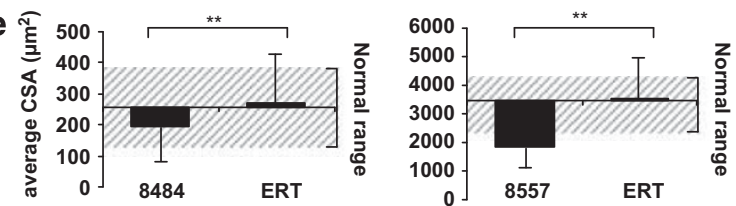

f

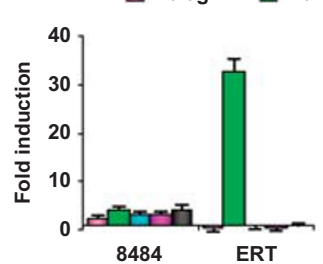

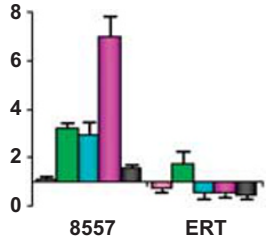

Figure 5 Comparison of pre- and post-ERT biopsies. (a) Immunoblot analysis of LC3, p62 and the loading control (GAPDH). ERT - : pre-ERT biopsy; ERT + : post-ERT biopsy; (b) H\&E staining and p62 immunohistochemistry. ERT: post-ERT biopsy. Bar: $40 \mu \mathrm{m}$. (c) CSA of fibers showing p62 aggregates (p62 aggr + ) compared with fibers without p62 aggregates ( $\mathrm{p} 62 \mathrm{aggr}-$ ) and vacuolated (vac + ) compared with non-vacuolated (vac - ) fibers, relative to healthy age- and sex-matched controls (mean CSA of infantile-onset controls: $258 \pm 124 \mu \mathrm{m}^{2}$; mean CSA of adult male controls: $\left.3446 \pm 950 \mu \mathrm{m}^{2}\right)$. The percentages of p62-positive, negative, vacuolated and non-vacuolated fibers are reported in the bars. ${ }^{* \star} P<0.0001$; ns: not significant. $n>200$ fibers measured. (d) CSA ratio of p62-aggregates positive/negative and vacuolated/non-vacuolated fibers. (e) Average CSA of all the muscle fibers. ${ }^{*} P<0.0001$. (f) qRT-PCR analysis. Fold induction is compared with the age-matched control and normalized to GAPDH

that these analyses are confounding in this patient because myofibers' morphology was disrupted by the massive vacuolization (Figure 5b). Indeed, MuRF1 expression in the infantile patient was tremendously induced in the second biopsy (Figure 5f).

Autophagy affects the maturation of GAA. To better understand the contribution of autophagy to GAA maturation and to muscle pathology, we monitored the effects of autophagy induction/inhibition in myotubes from GSDII patients and controls. To mimic Pompe disease, we used choloroquine to block lysosomal function. To activate autophagy, we used starvation alone or starvation together with rapamycin treatment, an $\mathrm{mTOR}$ inhibitor able to induce autophagosome formation. We monitored the presence/ absence of vacuoles in patients' myotubes before and after treatments. Myotubes from patients displayed vacuolization, while control did not show any vacuole unless they were treated with chloroquine. Interestingly, autophagy induction reduced the vacuolization observed in patients' myotubes (Figure 6a). Measurements of autophagy flux by using different lysosomal inhibitors confirmed that starvation and rapamycin treatments successfully activated autophagylysosome system in patients' myotubes (Supplementary 
a
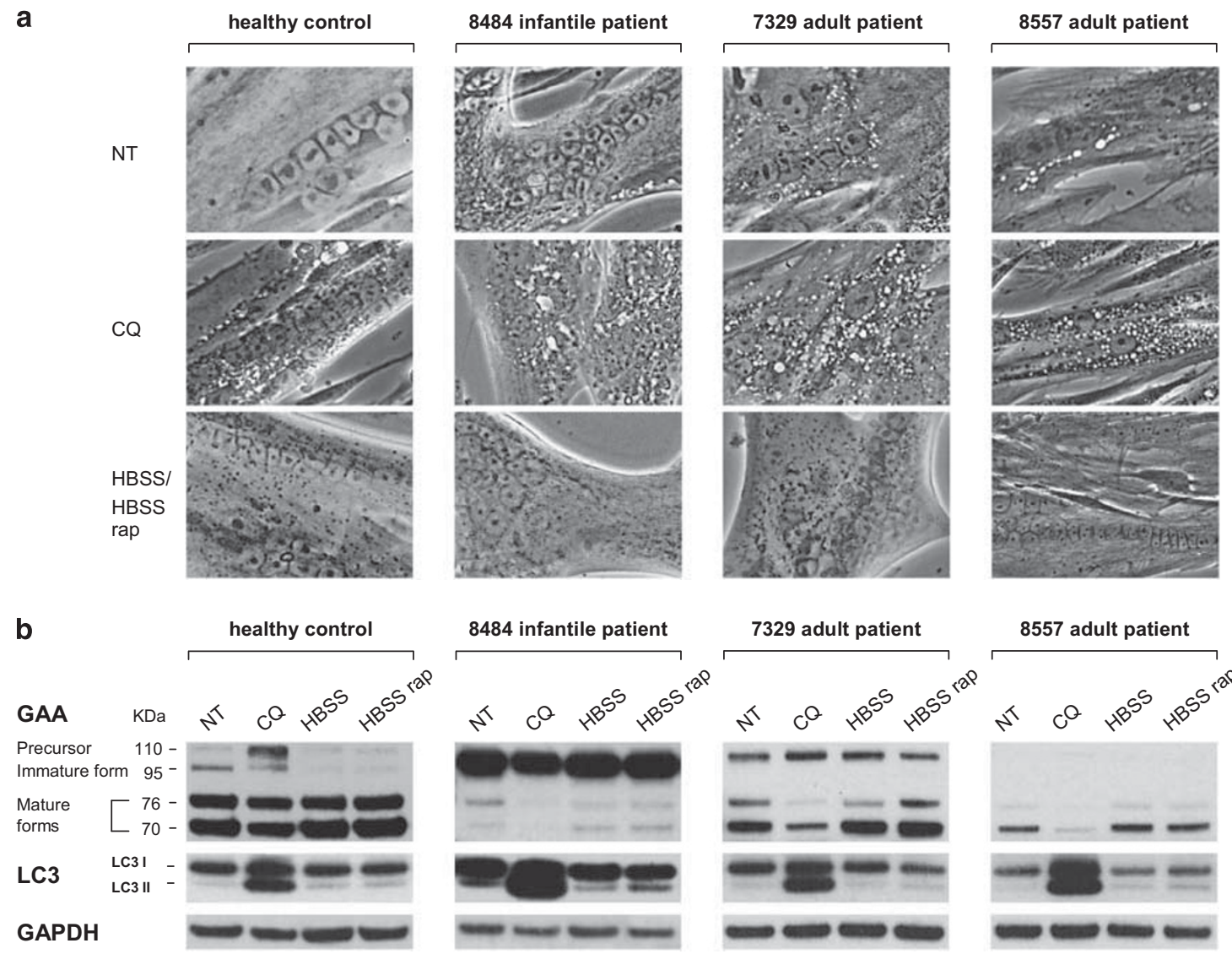

C

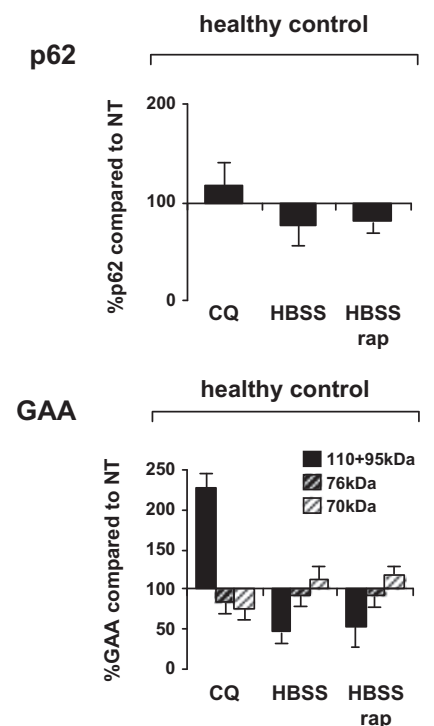

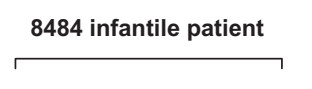
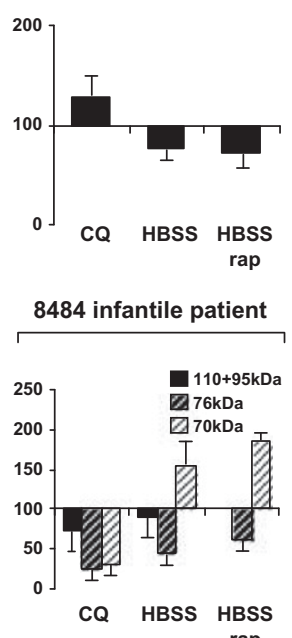
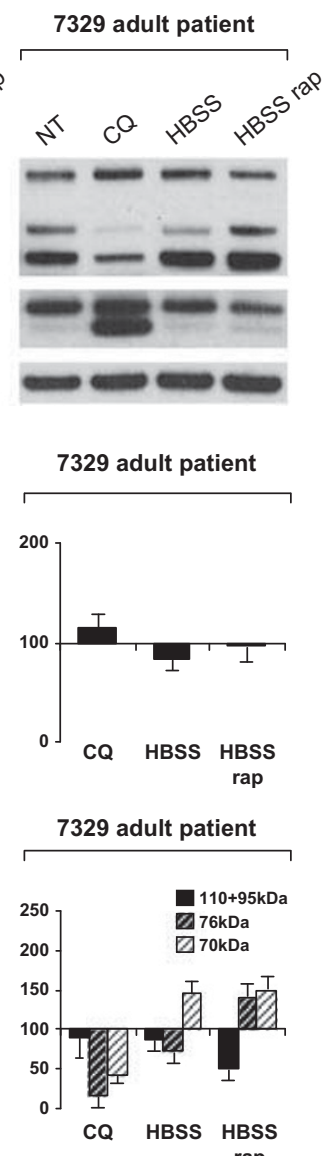

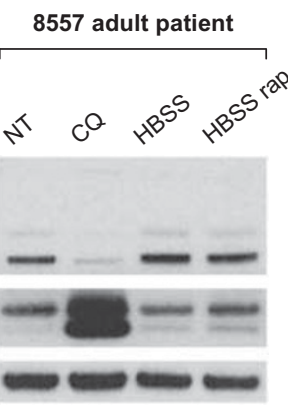

8557 adult patient
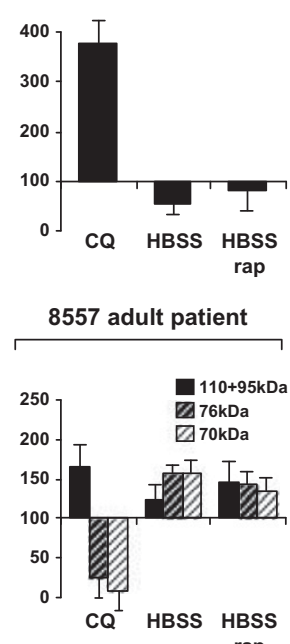

Figure 6 Induction/inhibition of autophagy in cultured healthy and patients' myotubes: autophagy enhances GAA processing into mature lysosomal forms. (a) Bright-field images of myotubes. NT: untreated; CQ: chloroquine-treated ( $50 \mu \mathrm{M} \mathrm{CQ}$ for $20 \mathrm{~h}$ in full medium); HBSS: starvation medium (HBSS for $40 \mathrm{~h}$ ); HBSS rap: starvation medium with rapamycin (HBSS with $0.3 \mu \mathrm{M}$ rapamycin for $40 \mathrm{~h}$ ). (b) Immunoblot analysis of GAA, LC3 and the loading control (GAPDH). NT: untreated; CQ: chloroquine-treated; HBSS: starvation medium; HBSS rap: starvation medium with rapamycin. (c) Densitometric analysis of p62 and GAA levels from different blots. Expression of inactive and active GAA forms ( 110 and $95 \mathrm{kDa}$ : inactive precursor and immature forms, respectively; 76 and $70 \mathrm{kDa}$ : mature lysosomal forms) in different conditions (autophagy inhibition: CQ; autophagy induction: HBSS and HBSS rap) compared with untreated myotubes (NT)

Figure S5).We then monitored the maturation of the GAA enzyme under these conditions. The myotubes obtained from infantile patient reproduced the same pattern of GAA species seen in the muscle biopsy, where the precursor protein was the most abundant one. Adult-derived myotubes displayed different mature forms of GAA. Our morphological and biochemical findings suggest that myotubes recapitulate the in-vivo conditions of GAA maturation. Induction of 
autophagy enhanced GAA maturation, whereas chloroquine produced the opposite effect (Figure 6b). Quantification of western blots confirmed the positive effect of autophagy on GAA processing, with a reduction in the immature form and enrichment of mature $76-70 \mathrm{kDa}$ forms (Figure $6 \mathrm{c}$ ). Consistently, choloroquine caused an accumulation of the $110 \mathrm{kDa}$ precursor in normal myotubes and disappearance or reduction of the $76-70 \mathrm{kDa}$ forms in patient-derived myotubes.

\section{Discussion}

Skeletal muscles of GSDII patients are characterized by a progressive vacuolization. The hallmark of this disorder is the presence of swollen, glycogen-filled lysosomes and the massive accumulation of autophagic debris, greatly contributing to the development of muscle weakness. However, the buildup of autophagosomes appears in myofibers when the autophagic system has already been compromised. The accumulation of autophagosomes has an important impact on cellular trafficking and signaling. Indeed, autophagosomes participate in the maturation of late endosomes, while p62 is critical for the modulation of important signaling molecules such as NFKB, NRF2 and PKC. Therefore, it is still unclear which is the role of autophagy-lysosome in GSDII patients when this system is still functioning. Indeed, the animal models of Pompe disease do not clarify this issue because inhibition or activation of autophagy neither ameliorates nor worsens the phenotype of GAA-KO mice. ${ }^{10,11}$ Surprisingly, glycogen levels also seem to be irrelevant to disease progression. Overexpression of $\mathrm{PGC} 1 \alpha$ greatly increases autophagic flux and glycogen overload in the muscle of GAA$\mathrm{KO}$ mice. However, it does not aggravate the muscle phenotype or affect disease progression. ${ }^{16}$ What animal models have shown is that there is a clear correlation between autophagosome accumulation and inefficiency of ERT treatment. This is not surprising because most of the cellular trafficking, including endosomal trafficking, uses proteins and lipids that also participate in autophagy system. Autophagosome accumulation can induce the sequestration of the factors that are also required for endosomal and vesicular trafficking and therefore, for recombinant GAA uptake and delivery to lysosomes.

Another important issue is whether early- and late-onset GSDII disorders share the same pathogenetic mechanisms of muscle weakness. Because GAA-KO mice completely lack the GAA enzyme, they are not the best model for understanding the mechanisms of muscle wasting when there is a partial defect in enzyme activity, as in late-onset GSDII. For these reasons, we have studied 17 muscle biopsies of 12 patients with different phenotypes, ranging from the most severe infantile-onset to the milder late-onset forms of the disease.

The effects of autophagic impairment on disease progression were monitored using the presence of p62-containing protein aggregates as a marker of autophagic flux inhibition. Indeed, when we specifically inhibited autophagy in muscle by a genetic approach, it resulted in accumulation of $\mathrm{p} 62$ protein and appearance of p62-positive aggregates. ${ }^{12}$
We found that p62 aggregate-positive and vacuolated fibers are more atrophic than surrounding p62 aggregate-negative and non-vacuolated fibers, both in infantile and in late-onset cases. The fact that p62-positive aggregates correlate with myofiber atrophy suggests that the alteration of autophagy system contributes to atrophy and weakness. The comparison of gene expression with morphometric analysis revealed a correlation between atrophy and MuRF1, but not Atrogin-1, expression.

Impaired autophagy was observed in infantile patients, where the presence of huge vacuoles in the majority of fibers coexisted with large amounts of autophagic debris, compromising the myofiber structure. Infantile patients showed accumulation of LC3II, p62 and aggregates. Altogether, these features confirm an impairment of the autophagic flux. Our results indicate that the Pompe patients constitute a homogeneous group for both clinical severity and autophagic impairment. We found that both vacuolated and p62-positive fibers showed a significant reduction of myofiber size, being the aggregate-positive fibers the most atrophic.

The correlation between autophagic impairment and muscle atrophy was particularly evident in late-onset patients. This group of patients showed heterogeneous muscle impairment and clinical outcomes and presented different degrees of autophagic impairment. The patients that showed a normal autophagic flux displayed a mild muscle pathology. Furthermore, the analyses of biopsies taken at different time points confirmed that disease progression correlates to autophagy impairment and atrophy. These findings suggest that autophagy acts as a protective mechanism during the early stages of the disease.

Indeed, our and other studies have found that autophagy inhibition in adult muscle results in muscle atrophy and weakness. ${ }^{12,17}$ Thus, late-onset disease progression may be physiologically accelerated by the inhibition of the autophagic flux. ERT was shown to re-establish the autophagic flux in muscle of a late-onset patient, resulting in an increase in muscle mass and in GAA processing and maturation. Furthermore, this increase was sufficient to downregulate the expression of atrogenes and to significantly enhance glycogen clearance. These beneficial effects on the autophagic flux and on muscle atrophy did not occur in the lately treated infantile patient. The fibers of this patient were too severely compromised by autophagic buildup and glycogen. Conversely, the early treated infantile patient displayed beneficial effects on autophagic flux, muscle mass and glycogen clearance, in agreement also with recent therapeutic trials, which indicate that an early therapeutic intervention prevents extensive muscle damage. ${ }^{15}$ These findings suggest that when the autophagic flux is greatly compromised also ERT is inefficient in restoring the flux and in improving muscle morphology and function. A possible explanation is that the autophagy machinery is required for an efficient uptake, maturation and delivery to lysosomes of recombinant GAA.

To test the hypothesis that the induction of autophagy could actually promote GAA maturation, we studied the effect of autophagy induction versus lysosomal inhibition in myotubes of control and GSDII patients. Morphological analyses confirmed that human myotubes, contrary to murine myotubes, ${ }^{18}$ are a reliable system to reproduce the GSDII 
pathology. The biochemical analyses confirmed that enhanced GAA maturation in both healthy and diseased myotubes depends on the autophagic flux.

In conclusion, our results suggest that autophagy may play an important role in the pathogenesis of both infantile and late-onset forms of GSDII, that could be protective against disease progression and atrophy. ERT, when started early, is successful in downregulating atrophy, possibly by partially restoring the physiological autophagic flux. Exhaustion of the autophagic flux induces upregulation of MuRF1, that leads to muscle atrophy and weakness.

\section{Materials and Methods}

Patients and muscle biopsies. We selected 12 GSDII cases (Supplementary Table S1) diagnosed following clinical examination, muscle biopsy histopathology, measurement of GAA activity in muscle and identification of mutations in the GAA gene. ${ }^{14}$ Muscle biopsies were obtained with informed consent and immediately processed by freezing in liquid nitrogen for histopathology and biochemical analysis and stored under liquid nitrogen for subsequent cell culture. Experiments were approved by the local ethics committee at the University of Padova. As normal controls, we used muscle biopsy specimens from patients who were free of any muscle disease and had normal creatine kinase levels. We selected age- and sex-matched controls for the morphometric analysis and age-matched controls for the immunoblotting and gene expression analyses. In our study, three GSDII patients were affected by the infantile-onset form of the disease, two had the childhood-onset form and the remaining had the adult-onset form. Among infantile patients, one (8668) received early-ERT treatment and then underwent biopsy.

Five cases (one infantile-onset, four adult-onset) underwent two muscle biopsies during the disease course. In three adult-onset cases, a second biopsy was taken after 6 (5638 and 5639) or 9 (4512) years of disease progression. In one infantileonset (8484) and one adult-onset (8557) case, the second biopsy followed, respectively, 16 and 6 months of ERT with recombinant human enzyme (Myozyme, Genzyme Corporation, Framingham, MA, USA).

Recombinant human GAA. RhGAA is administered as $110 \mathrm{kDa}$ precursor (Myozyme, Genzyme Corporation) $20 \mathrm{mg} / \mathrm{kg}$ every other week.

Immunoblotting. Thirty $10 \mu \mathrm{m}$ thick fresh-frozen sections of muscle biopsies were lysed in a buffer containing $50 \mathrm{mM}$ Tris, $\mathrm{pH} 7.5,150 \mathrm{mM} \mathrm{NaCl}, 10 \mathrm{mM} \mathrm{MgCl}_{2}$, $0.5 \mathrm{mM}$ DTT, $1 \mathrm{mM}$ EDTA, $10 \%$ glycerol, $2 \%$ SDS, $1 \%$ Triton X-100 and protease inhibitors (Complete Protease Inhibitor Cocktail, Roche SpA, Milan, Italy). Myotube protein extracts were obtained by lysing cells with $50 \mathrm{mM}$ Tris, $\mathrm{pH} 7.5$, $100 \mathrm{mM} \mathrm{NaCl}, 5 \mathrm{mM} \mathrm{MgCl}_{2}, 1 \mathrm{mM}$ DTT, $10 \%$ glycerol, $2 \%$ SDS, $0.5 \%$ Triton X-100 and protease inhibitors. The samples were immunoblotted as previously described ${ }^{19}$ and visualized with the chemiluminescent substrate ECL (GE Healthcare, Milan, Italy). When necessary, we stripped and reprobed the membranes. The stripping buffer consisted of $25 \mathrm{mM}$ glycine- $\mathrm{HCl}, \mathrm{pH} 2$ and $1 \%$ SDS. The antibody to LC3 (LC3-5F10) was from NanoTools Antikörpertechnik GmbH \& Co. KG (Teningen, Germany); the antibody to p62 (GP62-C) was from Progen Biotechnik GmbH (Heidelberg, Germany) and the antibody to GAA (mouse monoclonal antibody against the whole protein) was provided by Genzyme Corporation. Glyceraldehyde 3-phosphate dehydrogenase (GAPDH) (Abcam Ltd, Cambridge, UK) was used as loading control. We carried out densitometric quantification from multiple gels for each experiment using the ImageJ software (US National Institutes of Health, Bethesda, MD, USA)

Histology, fluorescence microscopy and morphometry. Cryosections of muscle biopsies were stained with hematoxylin-eosin (H\&E) and anti-p62. For histology, non-overlapping, random fields were imaged using a $\times 20$ objective (Zeiss Axioskop microscope, Gottingen, Germany), a Photometrics CoolSnap camera and the Roper Scientific Imaging Software (Ottobrunn, Germany). For immunofluorescence (IF), muscle cryosections were collected on Superfrost slides, fixed with $4 \%$ paraformaldehyde (PFA), treated with $0.1 \%$ Triton, incubated in blocking solution (0.5\% BSA, $10 \%$ horse serum in PBS) for $20 \mathrm{~min}$, and then incubated overnight at $4{ }^{\circ} \mathrm{C}$ with antibodies (the same used for immunoblotting, see above). Appropriate secondary fluorescent antibodies (Alexa-Fluor, Invitrogen,
Paisley, UK) were used. Slides were mounted using Vectashild medium with DAPI stain (Vector, Burlingame, CA, USA) and examined on a fluorescence Leica DM5000B microscope (Leica Microsystems Srl, Milan, Italy). In some cases, we performed also PAS staining and a double staining with anti-p62 and anti-LC3 or anti-caveolin-3 (cav3), incubating cryosections with the two antibodies sequentially at $4^{\circ} \mathrm{C}$ overnight, as above. Cav3 antibody (Transduction Lab, Lexington, KY, USA) was used to label both sarcolemmal and vacuolar membranes in adults. ${ }^{14}$

We calculated the percentage of vacuolated fibers, defined as those presenting either diffuse or scattered intracytoplasmic vacuoles, and of p62-positive fibers, defined as those presenting either diffuse or scattered intracytoplasmic staining. Fiber CSA was measured using ImageJ. For patients and controls, we calculated the total mean CSA by measuring all muscle fibers in the examined sections, the mean CSA of fibers with and without p62-positive protein aggregates and the mean CSA of vacuolated and non-vacuolated fibers. At least 200 fibers were measured in each biopsy. Mean reference fiber CSA in healthy controls was $258 \pm 124 \mu \mathrm{m}^{2}$ for infants $\left(n=3,5\right.$-18 months), $395 \pm 100 \mu \mathrm{m}^{2}$ for 4-year-old children $(n=3)$; $681 \pm 193 \mu \mathrm{m}^{2}$ for 7 -year-old children $(n=3) ; 2419 \pm 786 \mu \mathrm{m}^{2}$ for women $(n=6$, $32-54$ years) and $3446 \pm 950 \mu \mathrm{m}^{2}$ for men $(n=3 ; 18-28$ years).

Gene expression analyses. Total RNA was isolated from muscle biopsies with the Trizol reagent and treated with DNase I (Invitrogen) according to the manufacturer's protocol. The yield and purity of the extracted total RNA were determined using a Nanodrop spectrophotometer (Thermo Scientific, Austin, TX, USA). cDNA was generated with the iScript CDNA Synthesis Kit (Bio-Rad, Hercules, CA, USA) and analyzed by real-time PCR on an MJ Mini Opticon Thermal Cycler (Bio-Rad) using the IQ SYBR green super mix. Results were normalized to GAPDH using the $2^{-\Delta \Delta C T}$ calculation method. Primers specific for Bnip3 (RefSeq NM 004052), BECN1 (RefSeq NM 003766) and p62 (RefSeq NM_003900) were designed with the Primer3 software (http://frodo.wi.mit.edu/ primer3/). Previously published primer sequences were used for Atrogin-1, MuRF1 and GAPDH. ${ }^{20,21}$

Cell culture. Primary myoblasts from a healthy control and three GSDII patients $(8484,7329$ and 8557$)$ were obtained from fresh greatest gluteal, vastus lateralis and quadriceps femoris biopsies, respectively. The myoblasts of the healthy control were provided by Myosix (Paris Cedex, France). All samples were cultured until passage 3 at Myosix, and then in Ham F14 medium plus 20\% fetal bovine serum (FBS; Euroclone, Milan, Italy) with $1 \%$ insulin (Gibco, Invitrogen, Milan, Italy). At $70 \%$ confluence, myoblasts were induced to differentiate by lowering the FBS concentration to $2 \%$. Cells were maintained in differentiation medium for 5 days. Then, myotubes were treated with chloroquine (50 $\mu \mathrm{m}$; SigmaAldrich Srl, Milan, Italy) for $20 \mathrm{~h}$ or switched to a starvation medium (HBSS: Hank's-balanced salt solution, Gibco, Invitrogen) with or without rapamycin $(0.3 \mu \mathrm{m}$; LC Laboratories, Woburn, MA, USA) for $40 \mathrm{~h}$. Additional flux experiments were done with bafilomycin A (200 nM; Sigma-Aldrich Srl) and E64d $(10 \mu \mathrm{g} / \mathrm{ml}$; Sigma-Aldrich Srl) with pepstatin A $(50 \mu \mathrm{g} / \mathrm{ml}$; Sigma-Aldrich Srl) in full and starvation media (HBSS and HBSS with rapamycin) as inhibitors and with starvation media as inducers (6 and $12 \mathrm{~h}$ of incubation). Bright-field images of myotubes were collected using a Zeiss IM35 microscope equipped with a standard camera. After imaging the differentiation state of each sample, we solubilized the cells in lysis buffer for western blotting, as described above.

Statistical analysis. Data were expressed as mean values \pm S.D. Differences between groups were assessed using analysis of variance (Student's unpaired $t$-test) and linear regression analysis. We considered a $P$-value $<0.05$ to be significant.

\section{Conflict of Interest}

The authors declare no conflict of interest.

Acknowledgements. We gratefully acknowledge B Dalle from Myosix for providing and culturing some of the myoblasts and Genzyme for the anti-GAA antibody. This work was supported by grants from AFM (14199) to AN, from Telethon-Italy (GTB07001-DR) and Eurobiobank network (QLRT2001-027769) to CA, from AFM (15504), from Telethon-ltaly (TCP04009) and from ERC (MyoPHAGY, No: 282310) to MS. 
1. Mizushima N, Levine B, Cuervo AM, Klionsky DJ. Autophagy fights disease through cellular self-digestion. Nature 2008; 451: 1069-1075.

2. Mammucari C, Milan G, Romanello V, Masiero E, Rudolf R, Del Piccolo $P$ et al. FoxO3 controls autophagy in skeletal muscle in vivo. Cell Metab 2007; 6: 458-471.

3. Engel AG, Hirschhorn R, Huie ML. Acid maltase deficiency. In: Engel AG, Franzini-Armstrong C (eds). Myology. McGraw-Hill Press: New York, 2004, pp 1559-1586.

4. Nishino I, Fu J, Tanji K, Yamada T, Shimojo S, Koori T et al. Primary LAMP-2 deficiency causes X-linked vacuolar cardiomyopathy and myopathy (Danon disease). Nature 2000; 406: $906-910$.

5. Kalimo H, Savontaus ML, Lang H, Paljärvi L, Sonninen V, Dean PB et al. X-linked myopathy with excessive autophagy: a new hereditary muscle disease. Ann Neurol 1988 23: 258-265.

6. Ramachandran N, Munteanu I, Wang P, Aubourg P, Rilstone JJ, Israelian N et al. VMA21 deficiency causes an autophagic myopathy by compromising V-ATPase activity and lysosomal acidification. Cell 2009; 137: 235-246.

7. Stauber WT, Hedge AM, Trout JJ, Schottelius BA. Inhibition of lysosomal function in red and white skeletal muscles by chloroquine. Exp Neurol 1981; 71: 295-306.

8. Raben N, Takikita S, Pittis MG, Bembi B, Marie SK, Roberts A et al. Deconstructing Pompe disease by analyzing single muscle fibers: to see a world in a grain of sand. Autophagy 2007; 3: 546-552.

9. Raben N, Baum R, Schreiner C, Takikita S, Mizushima N, Ralston E et al. When more is less: excess and deficiency of autophagy coexist in skeletal muscle in Pompe disease. Autophagy 2009; 5: 111-113.

10. Raben N, Schreiner C, Baum R, Takikita S, Xu S, Xie T et al. Suppression of autophagy permits successful enzyme replacement therapy in a lysosomal storage disorder-murine Pompe disease. Autophagy 2010; 6: 1078-1089.

11. Raben N, Hill V, Shea L, Takikita S, Baum R, Mizushima N et al. Suppression of autophagy in skeletal muscle uncovers the accumulation of ubiquitinated proteins and their potential role in muscle damage in Pompe disease. Hum Mol Genet 2008; 17: 3897-3908.
12. Masiero E, Agatea L, Mammucari $C$, Blaauw B, Loro E, Komatsu $M$ et al. Autophagy is required to maintain muscle mass. Cell Metab 2009; 10: 507-515.

13. Lecker SH, Jagoe RT, Gilbert A, Gomes M, Baracos V, Bailey J et al. Multiple types of skeletal muscle atrophy involve a common program of changes in gene expression. FASEB J 2004; 18: 39-51.

14. Nascimbeni AC, Fanin M, Tasca E, Angelini C. Molecular pathology and enzyme processing in various phenotypes of acid maltase deficiency. Neurology 2008; 70: 617-626.

15. Chien YH, Lee NC, Thurberg BL, Chiang SC, Zhang XK, Keutzer J et al. Pompe disease in infants: improving the prognosis by newborn screening and early treatment. Pediatrics 2009; 124: e1116-e1125.

16. Takikita S, Schreiner C, Baum R, Xie T, Ralston E, Plotz PH et al. Fiber type conversion by PGC-1 $\alpha$ activates lysosomal and autophagosomal biogenesis in both unaffected and Pompe skeletal muscle. PLoS One 2010; 5: e15239.

17. Salminen A, Kaarniranta K. Regulation of the aging process by autophagy. Trends $\mathrm{Mol}$ Med 2009; 15: 217-224.

18. Takikita S, Myerowitz R, Schreiner C, Baum R, Raben N, Plotz PH. The values and limits of an in vitro model of Pompe disease: the best laid schemes o' mice an' men. Autophagy 2009; 5: 729-731.

19. Sandri M, Sandri C, Gilbert A, Skurk C. Calabria E, Picard A et al. Foxo transcription factors induce the atrophy-related ubiquitin ligase atrogin-1 and cause skeletal muscle atrophy. Cell 2004; 117: 399-412.

20. Léger $B$, Vergani L, Sorarù $G$, Hespel $P$, Derave $W$, Gobelet $C$ et al. Human skeletal muscle atrophy in amyotrophic lateral sclerosis reveals a reduction in Akt and an increase in atrogin-1. FASEB 2006; 20: 583-585.

21. Vandesompele J, De Preter K, Pattyn F, Poppe B, Van Roy N, De Paepe A et al. Accurate normalization of real-time quantitative RT-PCR data by geometric averaging of multiple internal control genes. Genome Biol 2002; 3 (research0034). $1-11$.

Supplementary Information accompanies the paper on Cell Death and Differentiation website (http://www.nature.com/cdd) 